\title{
4D Hyperspherical Harmonic (HyperSPHARM) Representation of Multiple Disconnected Brain Subcortical Structures
}

\author{
Ameer Pasha Hosseinbor ${ }^{1}$, Moo K. Chung ${ }^{1}$, Stacey M. Schaefer ${ }^{1}$, \\ Carien M. van Reekum ${ }^{2}$, Lara Peschke-Schmitz ${ }^{1}$, Matt Sutterer ${ }^{1}$, \\ Andrew L. Alexander ${ }^{1}$, and Richard J. Davidson ${ }^{1}$ \\ 1 University of Wisconsin-Madison, USA \\ 2 University of Reading, UK \\ hosseinbor@wisc.edu
}

\begin{abstract}
We present a novel surface parameterization technique using hyperspherical harmonics (HSH) in representing compact, multiple, disconnected brain subcortical structures as a single analytic function. The proposed hyperspherical harmonic representation (HyperSPHARM) has many advantages over the widely used spherical harmonic (SPHARM) parameterization technique. SPHARM requires flattening 3D surfaces to $3 \mathrm{D}$ sphere which can be time consuming for large surface meshes, and can't represent multiple disconnected objects with single parameterization. On the other hand, HyperSPHARM treats 3D object, via simple stereographic projection, as a surface of $4 \mathrm{D}$ hypersphere with extremely large radius, hence avoiding the computationally demanding flattening process. HyperSPHARM is shown to achieve a better reconstruction with only 5 basis compared to SPHARM that requires more than 441 .
\end{abstract}

\section{Introduction}

Many shape modeling frameworks in computational anatomy assume topological invariance between objects and are not applicable for objects with different or changing topology. There are numerous such examples from longitudinal child development to cancer growth. For example, an infant may have about 300-350 bones at birth but an adult has 206 bones. These bones fuse together as the infant grows. This type of topological difference and changes cannot be incorporated directly into the processing and analysis pipeline with existing shape models that assume topological invariance and mainly work on a single connected component. The difficulty is mainly caused by the lack of a single, coherent mathematical parameterization for multiple, disconnected objects.

Probably the most widely applied shape parameterization technique for cortical structures is the spherical harmonic (SPHARM) representation [47/10, which has been mainly used as a data reduction technique for compressing global shape features into a small number of coefficients. The main global geometric features are encoded in low degree coefficients while the noise will be in high degree spherical harmonics. The method has been used to model various brain structures such as ventricles [7, hippocampi [10] and cortical surfaces 
4]. SPHARM, however, can't represent multiple disconnected structures with a single parameterization. Since SPHARM requires a smooth map from surfaces to a 3D sphere, various computationally intensive surface flattening techniques have been proposed: diffusion mapping [4], conformal mapping [1], and area preserving mapping [7. The surface flattening is used to parameterize the surface using two spherical angles. The angles serve as coordinates for representing the surface using spherical harmonics. Then the surface coordinates can be mapped onto the sphere and each coordinate is represented as a linear combination of spherical harmonics.

Note that a $3 \mathrm{D}$ volume is a surface in $4 \mathrm{D}$. By performing simple stereographic projection on a $3 \mathrm{D}$ volume, it is possible to embed the $3 \mathrm{D}$ volume onto the surface of a $4 \mathrm{D}$ hypersphere, which bypasses the difficulty of flattening 3D surface to 3D sphere. Extending the concept further, any two or more disconnected 3D objects can be projected onto a single connected surface in $4 \mathrm{D}$ hypersphere. Then the disconnected 3D objects can be represented as the linear combination of $4 \mathrm{D}$ hyperspherical harmonics (HSH), which are the multidimensional analogues of the 3D spherical harmonics.

The HSH have been mainly confined to quantum chemistry, where their utility first became evident with respect to solving the Schrödinger equation for the hydrogen atom. It had been solved in position-space by Schrödinger, himself, but not in momentum-space, which is related to position-space via the Fourier transform. Sometime later, V. Fock solved the Schrödinger equation for the hydrogen atom directly in momentum-space. In his classic paper [6], Fock stereographically projected $3 \mathrm{D}$ momentum-space onto the surface of a $4 \mathrm{D}$ unit hypersphere, and after this mapping was made, he was able to show that the eigenfuctions were the 4D HSH. Recently, the HSH have been utilized in a wider array of fields than just quantum chemistry, including computer graphics visualization [3] and crystallography [9]. However, as of yet, they have remained elusive for medical imaging.

In this paper, following the approach of Fock, we model multiple disconnected $3 \mathrm{D}$ objects in terms of the 4D HSH by stereographically projecting each object's surface coordinates onto a 4D hypersphere, and label such a representation HyperSPHARM. Significantly, we show that HyperSPHARM can better reconstruct such objects than SPHARM using just a few basis functions. The method is applied to modeling disconnected brain subcortical structures, specifically the left and right hippocampus and amygdala.

\section{Methods}

\subsection{D Hyperspherical Harmonics}

Consider the $4 \mathrm{D}$ unit hypersphere $S^{3}$ existing in $\mathbb{R}^{4}$. The Laplace-Beltrami operator on $S^{3}$ is defined as $\Delta_{S^{3}}=\frac{1}{\sin ^{2} \beta} \frac{\partial}{\partial \beta} \sin ^{2} \beta \frac{\partial}{\partial \beta}+\frac{1}{\sin ^{2} \beta} \Delta_{S^{2}}$, where $\Delta_{S^{2}}$ is the Laplace-Beltrami operator on the unit sphere $S^{2}$. The eigenfuctions of $\Delta_{S^{3}}$ are the $4 \mathrm{D}$ HSH $Z_{n l}^{m}(\beta, \theta, \phi): \Delta_{S^{3}} Z_{n l}^{m}=-l(l+2) Z_{n l}^{m}$. The $4 \mathrm{D}$ HSH are defined as [5] 
Table 1. List of a Few HSH

\begin{tabular}{|cl|}
$Z_{00}^{0}(\beta, \theta, \phi)=\frac{1}{\pi \sqrt{2}}$ & $Z_{10}^{0}(\beta, \theta, \phi)=\frac{\sqrt{2}}{\pi} \cos \beta$ \\
$Z_{11}^{-1}(\beta, \theta, \phi)=\frac{-\sqrt{2}}{\pi} \sin \beta \sin \theta \sin \phi$ & $Z_{11}^{0}(\beta, \theta, \phi)=\frac{\sqrt{2}}{\pi} \sin \beta \cos \theta$ \\
$Z_{11}^{1}(\beta, \theta, \phi)=\frac{-\sqrt{2}}{\pi} \sin \beta \sin \theta \cos \phi$ & $Z_{20}^{0}(\beta, \theta, \phi)=\frac{1}{\pi \sqrt{2}}\left(3-4 \sin ^{2} \beta\right)$ \\
$Z_{21}^{-1}(\beta, \theta, \phi)=\frac{-\sqrt{3}}{\pi} \sin 2 \beta \sin \theta \sin \phi$ & $Z_{21}^{0}(\beta, \theta, \phi)=\frac{\sqrt{3}}{\pi} \sin 2 \beta \cos \theta$ \\
\hline
\end{tabular}

$$
Z_{n l}^{m}(\beta, \theta, \phi)=2^{l+1 / 2} \sqrt{\frac{(n+1) \Gamma(n-l+1)}{\pi \Gamma(n+l+2)}} \Gamma(l+1) \sin ^{l} \beta C_{n-l}^{l+1}(\cos \beta) Y_{l}^{m}(\theta, \phi),
$$

where $(\beta, \theta, \phi)$ obey $(\beta \in[0, \pi], \theta \in[0, \pi], \phi \in[0,2 \pi]), C_{n-1}^{l+1}$ are the Gegenbauer (ultraspherical) polynomials, and $Y_{l}^{m}$ are the $3 \mathrm{D}$ spherical harmonics. The index $l$ denotes the degree of the HSH, $m$ is the order, and $n=0,1,2, \ldots$, and these three integers obey the conditions $0 \leq l \leq n$ and $-l \leq m \leq l$. The number of HSH corresponding to a given value of $n$ is $(n+1)^{2}$. The first few $4 \mathrm{D}$ HSH are shown in Table 1. The HSH form an orthonormal basis on the hypersphere, and the normalization condition reads

$$
\int_{0}^{2 \pi} \int_{0}^{\pi} \int_{0}^{\pi} Z_{n l}^{m}(\beta, \theta, \phi) Z_{n^{\prime} l^{\prime}}^{m^{\prime} *}(\beta, \theta, \phi) \sin ^{2} \beta \sin \theta d \beta d \theta d \phi=\delta_{n n^{\prime}} \delta_{l l^{\prime}} \delta_{m m^{\prime}}
$$

\subsection{D Stereographic Projection of 3D Surface Coordinates onto Hypersphere}

Consider a 3D finite, compact object (i.e. has no singularities) comprised of surface coordinates $\mathbf{s}=\left(s^{1}, s^{2}, s^{3}\right)$. In order to model the surface coordinates with the HSH, we need to map them onto a 4D hypersphere, which can be achieved via stereographic projection [6]. The surface coordinates in spherical space are $s^{1}=r \sin \theta \cos \phi, s^{2}=r \sin \theta \sin \phi$, and $s^{3}=r \cos \theta$, where $r=$ $\sqrt{\left(s^{1}\right)^{2}+\left(s^{2}\right)^{2}+\left(s^{3}\right)^{2}}$. Consider a $4 \mathrm{D}$ hypersphere of radius $p_{o}$, whose coordinates are defined as

$$
\begin{aligned}
& u_{1}=p_{o} \sin \beta \sin \theta \cos \phi \\
& u_{2}=p_{o} \sin \beta \sin \theta \sin \phi \\
& u_{3}=p_{o} \sin \beta \cos \theta \\
& u_{4}=p_{o} \cos \beta .
\end{aligned}
$$

The relationship between $\left(s^{1}, s^{2}, s^{3}\right)$ and $\left(u_{1}, u_{2}, u_{3}, u_{4}\right)$ is then

$$
u_{1}=\frac{2 p_{o}^{2} s^{1}}{r^{2}+p_{o}^{2}}, \quad u_{2}=\frac{2 p_{o}^{2} s^{2}}{r^{2}+p_{o}^{2}}, \quad u_{3}=\frac{2 p_{o}^{2} s^{3}}{r^{2}+p_{o}^{2}}, \quad u_{4}=\frac{p_{o}\left(r^{2}-p_{o}^{2}\right)}{r^{2}+p_{o}^{2}}
$$



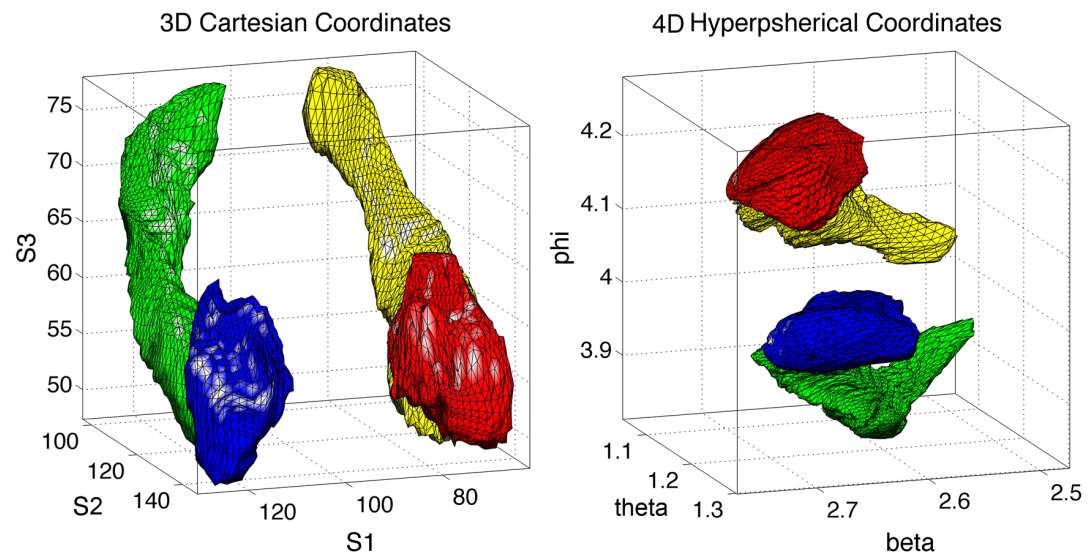

Fig. 1. The 3D subcortical structures (left) in the coordinates $\left(s^{1}, s^{2}, s^{3}\right)$ went through the $4 \mathrm{D}$ stereographic projection that resulted in conformally deformed structures (right) in the $4 \mathrm{D}$ spherical coordinates $(\beta, \theta, \phi)$. The $3 \mathrm{D}$ subcortical structure is then embedded on the surface of the $4 \mathrm{D}$ hypersphere with radius $p_{0}=2000$, which makes the surface of the hypersphere to be almost Euclidean.

According to Eq. (3) , the surface coordinate $(0,0,0)$ projects onto the south pole $\left(0,0,0,-p_{o}\right)$ of the hypersphere. As $r \rightarrow \infty$, the projection $\left(u_{1}, u_{2}, u_{3}, u_{4}\right)$ moves closer to the north pole $\left(0,0,0, p_{o}\right)$ of hypersphere. Thus, the north pole is not associated with any $\left(s^{1}, s^{2}, s^{3}\right)$, but gives us a way of envisioning infinity as a point. Eq. (3) establishes a one-to-one correspondence between the 3D volume and $4 \mathrm{D}$ hypersphere (Figure 1). The radius of the hypersphere $p_{o}$ controls the density of the projected surface coordinates onto the hypersphere's surface.

Stereographic projection exhibits two important properties. First, it is conformal, which means it preserves angles - the angles $(\theta, \phi)$ defining the 3D surface are preserved in $4 \mathrm{D}$ hyperspherical space. However, stereographic projection does not preserve volume; in general, the volume of a region in the $3 \mathrm{D}$ plane doesn't equal the volume of its projection onto the hypersphere.

\subsection{HSH Expansion of 3D Surface Coordinates}

Stereographically projecting a 3D object's surface coordinates onto a 4D hypersphere results in them existing along the hypersphere's surface. According to Fourier analysis, any square-integrable function defined on a sphere can be expanded in terms of the spherical harmonics. Thus, we can expand each coordinate component $s^{i}$, where $i=1,2,3$, in terms of the $4 \mathrm{D} \mathrm{HSH}$ :

$$
s_{p_{o}}^{i}(\beta, \theta, \phi) \approx \sum_{n=0}^{N} \sum_{l=0}^{n} \sum_{m=-l}^{l} C_{n l m}^{i} Z_{n l}^{m}(\beta, \theta, \phi),
$$



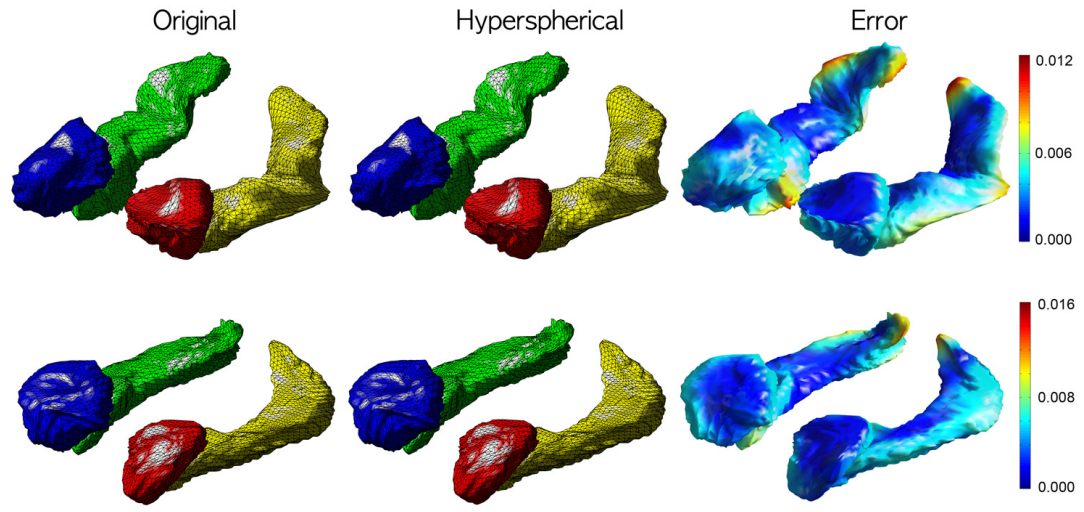

Fig. 2. Hyperspherical harmonic representation of amygdala and hippocampus surfaces for subject 10 and 60 . The vertex-wise reconstruction errors are also plotted. As expected, most errors are occurring near sharp peaks and corners.

where $s_{p_{o}}^{i}$ denotes the $i^{\text {th }}$ component of the surface coordinates $\mathbf{s}$ existing on hypersphere of radius $p_{o}$. The realness of the surface coordinates requires use of the real $\mathrm{HSH}$, and so we employ a modified real basis used in 8 ] for $Y_{l}^{m}$. For a given truncation order $N$, the total number of expansion coefficients is $W=(N+1)(N+2)(2 N+3) / 6$.

\subsection{Numerical Implementation}

Suppose each $s^{i}$ of our 3D surface consists of $M$ vertices. This is the total number of mesh vertices for all disconnected surfaces. The task then is to estimate each $s^{i}$ 's coefficients $C_{n l m}^{i}$ in Eq. (4) from the $M$ surface vertices.

Let $\Omega_{j}=\left(\beta_{j}, \theta_{j}, \phi_{j}\right)$ denote the hyperspherical angles at the $j$-th mesh vertex. Denote $\mathbf{s}^{i}$ as the $M \times 1$ vector representing each $s^{i}$ 's $M$ vertices, $\mathbf{C}^{i}$ the $W \times 1$ vector of unknown expansion coefficients $C_{n l m}^{i}$ for each $s^{i}$, and $\mathbf{A}$ the $M \times W$ matrix constructed with the HSH basis

$$
\mathbf{A}=\left(\begin{array}{cccccc}
Z_{00}^{0}\left(\Omega_{1}\right) & Z_{10}^{0}\left(\Omega_{1}\right) & Z_{11}^{-1}\left(\Omega_{1}\right) & Z_{11}^{0}\left(\Omega_{1}\right) & \cdots & Z_{N N}^{N}\left(\Omega_{1}\right) \\
\vdots & \vdots & \vdots & \vdots & \ddots & \vdots \\
Z_{00}^{0}\left(\Omega_{M}\right) & Z_{10}^{0}\left(\Omega_{M}\right) & Z_{11}^{-1}\left(\Omega_{M}\right) & Z_{11}^{0}\left(\Omega_{M}\right) & \cdots & Z_{N N}^{N}\left(\Omega_{M}\right)
\end{array}\right)
$$

Thus, the general linear system representing Eq. (4) is described by $\mathbf{s}^{i}=\mathbf{A} \mathbf{C}^{i}$. This system of over-determined equations is solved via linear least squares, yielding

$$
\widehat{\mathbf{C}^{i}}=\left(\mathbf{A}^{T} \mathbf{A}\right)^{-1} \mathbf{A}^{T} \mathbf{s}^{i}
$$

The reconstructed $s^{i}$ is $\widehat{\mathbf{s}^{i}}=\mathbf{A} \widehat{\mathbf{C}^{i}}$, and so our reconstructed $3 \mathrm{D}$ surface is defined by the $M \times 3$ matrix $\widehat{\mathbf{s}}=\left(\widehat{s^{1}}, \widehat{s^{2}}, \widehat{s^{3}}\right)$. The mean squared error (MSE) between 
Table 2. The mean squared error (MSE) and its standard deviation of reconstruction. MSE is computed over all mesh vertices and averaged over 69 subjects. Degree 2, 10 and 20 SPHARM representations require $3^{2}=9,11^{2}=121$ and $21^{2}=441$ basis functions, respectively. The reconstruction error of HSH expansion of order $N=1$ is substantially smaller than those of SPHARM, even though only 5 HSH basis functions are used.

\begin{tabular}{|l|l|l|l|c|}
\hline & SPHARM 2 & SPHARM 10 & SPHARM 20 & HSH 1 \\
\hline Left Amygdala & $1.08 \pm 0.17$ & $0.054 \pm 0.010$ & $0.022 \pm 0.005$ & $0.18 \pm 0.04 \times 10^{-5}$ \\
Right Amygdala & $0.60 \pm 0.11$ & $0.052 \pm 0.008$ & $0.023 \pm 0.003$ & $0.27 \pm 0.06 \times 10^{-5}$ \\
Left Hippocampus & $1.77 \pm 0.33$ & $0.127 \pm 0.026$ & $0.043 \pm 0.040$ & $0.90 \pm 0.20 \times 10^{-5}$ \\
Right Hippocampus & $1.08 \pm 0.17$ & $0.054 \pm 0.010$ & $0.022 \pm 0.005$ & $0.18 \pm 0.04 \times 10^{-5}$ \\
\hline
\end{tabular}

the original surface and the reconstructed surface can then be computed as $\operatorname{tr}\left[(\mathbf{s}-\widehat{\mathbf{s}})^{T}(\mathbf{s}-\widehat{\mathbf{s}})\right] / M$.

\section{$3 \quad$ Experimental Results and Applications}

We collected high-resolution T1-weighted inverse recovery fast gradient echo MRI in 124 contiguous $1.2-\mathrm{mm}$ axial slices $(\mathrm{TE}=1.8 \mathrm{~ms} ; \mathrm{TR}=8.9 \mathrm{~ms}$; flip angle $=10^{\circ} ; \mathrm{FOV}=240 \mathrm{~mm} ; 256 \times 256$ data acquisition matrix) of 69 middle-age and elderly adults ranging between 38 to 79 years (mean age $=58.0 \pm 11.4$ years) The data were collected as a part of a national study called MIDUS (Midlife in US; http://midus.wisc.edu) for the health and well-being in the aged population [12. There are 23 men and 46 women in the study. Brain tissues in the MRI scans were automatically segmented using Brain Extraction Tool (BET) 11 and trained raters manually segmented the parts of limbic system: amygdala and hippocampus. A nonlinear image registration using the diffeomorphic shape and intensity averaging technique with the cross-correlation as the similarity metric through Advanced Normalization Tools (ANTS) [2] was performed and the study specific template is constructed. The isosurfaces of the segmentation were extracted using the marching cube algorithm (Figure 2).

Four brain subcortical structures, specifically the left and right hippocampus and amygdala, were reconstructed for 69 subjects. For each structure, the HSH expansion parameters were $N=1$ and radius $p_{o}=2000$, which results in $W=$ $5 \mathrm{HSH}$ expansion coefficients for each $s^{i}$. So total of $15 \mathrm{HSH}$ coefficients can parameterize a single surface and 60 coefficients all four disconnected surfaces (i.e. left and right hippocampus and amygdala). Figure 2 shows the reconstructed HSH surfaces for two subjects. The length of the residual is computed and plotted on the reconstructed surfaces. As expected, the most reconstruction errors are found near peaks and corners.

\subsection{Comparison against SPHARM Representation}

We have compared the reconstruction errors between HSH and SPHARM representations. Hippocampus and amygdala surface meshes are flattened to a unit 

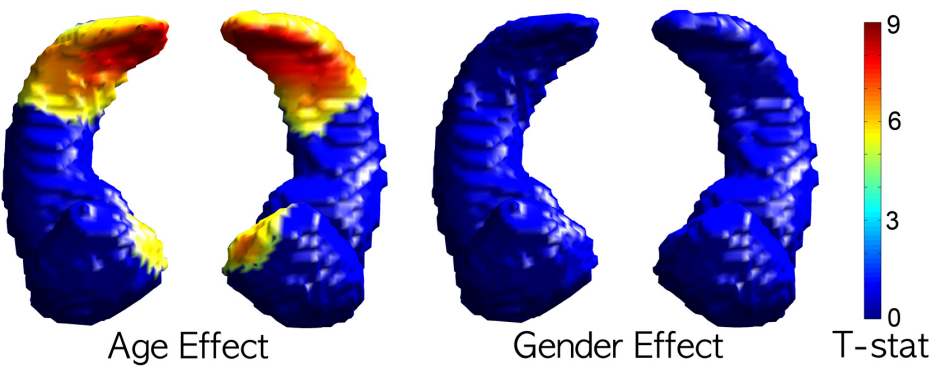

Fig. 3. The regions showing statistically significant age effect thresholded at $p<0.05$ (corrected). There is no gender effect.

sphere and resampled to a uniform grid along the sphere. Then degree 2, 10 and 20 SPHARM representations are constructed. The MSE of each reconstruction is computed within each surface and averaged over 69 subjects (Table 2). Its standard error is also computed over all 69 subjects. The degree $k$ SPHARM representations requires $(k+1)^{2}$ SPHARM basis functions.

The degree 2, 10 and 20 SPHARM representations require 9, 121 and 441 basis functions. Even with only 5 basis functions, HSH is achieving substantially low MSE compared with SPHARM reconstruction with 441 basis functions, demonstrating the superior efficiency in the HSH representation.

\subsection{Influence of Age and Gender}

HSH representations were obtained for hippocampus and amygdala surfaces of all 69 subjects. The representation behaves like surface smoothing technique where high frequency noise is removed as shown in Figure 2 The 69 reconstructed surfaces are then averaged to produce the population specific template. The 3D displacement vector field from the template to individual surface is taken as the response vector in the multivariate general linear model (MGLM) 4 and its $T$-statistic is computed and thresholded at $p<0.05$. The random field based multiple comparisons are performed to give stringent results. We have detected significant influence of age mainly on the tail regions of the hippocampus while there is no influence of gender on any of the structures.

\section{Conclusion and Discussion}

In this paper, we have presented a new analytic approach for representing multiple disconnected shapes using a single analytic function, which is a linear combination of HSH. The method is applied to parameterizing 4 disconnected subcortical structures (two amygdalae and two hippocampi) using only $60 \mathrm{HSH}$ coefficients. The resulting HSH coefficients are global and contain information about all four structures, so they do not provide any local shape information. 
Therefore, HyperSPHARM might be better suited to sparse techniques such as wavelets, which will be explored in future. Despite HSH being a global basis, by reconstructing surfaces at each voxel and using $\mathrm{HSH}$ as a way to filter out high frequency noise, it was possible to use HSH for local inference at vertex level as shown in our application. Although the individual image volumes are registered to a template using diffeomorphic warping [2, we might only need an affine registration to initially align the structures and simply match the coefficients as in SPHARM [4] but the issue is not explored here and left as a future study.

Acknowledgements. This research was supported by the National Institute of Aging (P01-AG20166) and National Institute on Mental Health (R01 MH043454). Seung-Goo Kim of Max Planck Institutes performed image registration.

\section{References}

1. Angenent, S., Hacker, S., Tannenbaum, A., Kikinis, R.: On the laplace-beltrami operator and brain surface flattening. IEEE Transactions on Medical Imaging 18, 700-711 (1999)

2. Avants, B., Epstein, C., Grossman, M., Gee, J.: Symmetric diffeomorphic image registration with cross-correlation: Evaluating automated labeling of elderly and neurodegenerative brain. Medical Image Analysis 12, 26-41 (2008)

3. Bonvallet, B., Griffin, N., Li, J.: 3D shape descriptors: 4D hyperspherical harmonics "An exploration into the fourth dimension". In: IASTED International Conference on Graphics and Visualization in Engineering, pp. 113-116 (2007)

4. Chung, M., Worsley, K., Brendon, M., Dalton, K., Davidson, R.: General multivariate linear modeling of surface shapes using SurfStat. NeuroImage 53, 491-505 (2010)

5. Domokos, G.: Four-dimensional symmetry. Physical Review 159, 1387-1403 (1967)

6. Fock, V.: Zur theorie des wasserstoffatoms. Z. Physik 98, 145-154 (1935)

7. Gerig, G., Styner, M., Jones, D., Weinberger, D., Lieberman, J.: Shape analysis of brain ventricles using spharm. In: MMBIA, pp. 171-178 (2001)

8. Koay, C.G., Ozarslan, E., Basser, P.J.: A signal transformational framework for breaking the noise floor and its applications in MRI. J. Magn. Reson. 197, 108-119 (2009)

9. Mason, J.K., Schuh, C.A.: Hyperspherical harmonics for the representation of crystallographic texture 56, 6141-6155 (2008)

10. Shen, L., Ford, J., Makedon, F., Saykin, A.: surface-based approach for classification of 3d neuroanatomical structures. Intelligent Data Analysis 8, 519-542 (2004)

11. Smith, S.: Fast robust automated brain extraction. Human Brain Mapping 17, 143-155 (2002)

12. Van Reekum, C., Schaefer, S., Lapate, R., Norris, C., Greischar, L., Davidson, R.: Aging is associated with positive responding to neutral information but reduced recovery from negative information. Social Cognitive and Affective Neuroscience 6, 177-185 (2011) 